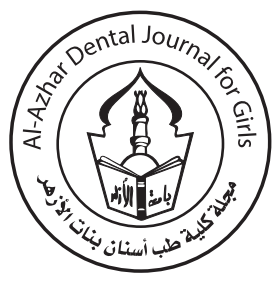

\title{
The Effect of Application of Forsterite Nanoparticles in Different Protocols on Permeability of Superficial and Deep Dentin
}

\author{
Sarah M. Mohamed ${ }^{1 *}$, Maha A. Niazy², Randa M. Hafez ${ }^{3}$ and Maha I. Elkorashy ${ }^{4}$
}

Codex : 42/1907

azhardentj@azhar.edu.eg

http://adjg.journals.ekb.eg

DOI: $10.21608 /$ adjg.2019.6896.1068

\begin{abstract}
Purpose: This study evaluated and compared the effect of application of Forsterite nanoparticles in different protocols on permeability of superficial and deep dentin at different time intervals. Materials and methods: thirty human molars were used to prepare 60 dentin discs, divided into three main groups $(n=20)(P) ;(P 1)$ : Forsterite nanoparticles alone, (P2): Forsterite nanoparticles added to an adhesive system and (P3): Forsterite nanoparticles incorporated in Glass Ionomer. The main groups were subdivided into two subgroups $(n=10)$ according to the dentin depth (D); (D1): superficial dentin and (D2): deep dentin. Permeability was measured at three time intervals (T); (T1) baseline, (T2) after one week and (T3) after four weeks. Three wt\% of forsterite nanoparticles were added to adhesive system and Glass ionomer. Teeth were sectioned to obtain superficial and deep dentin discs of; $1 \mathrm{~mm}( \pm 0.1 \mathrm{~mm})$ thickness Modified Pashly's split chamber was used to measure dentin permeability at baseline and after application of different protocols. Representative samples were assessed with ESEM and EDAX. Results: all protocols showed permeability reduction. (P2) showed the least permeability at T2 for superficial and deep dentin discs, (P1) showed the least permeability at T3 for both superficial and deep dentin discs and (P3) showed the least permeability reduction. Conclusion: it was concluded that Forsterite nanoparticles applied in different protocols can reduce dentin permeability in superficial and deep dentin. Superior dentin permeability reduction could be achieved after one week of using Forsterite nanoparticles added to the adhesive system and four weeks after using Forsterite nanoparticles alone.
\end{abstract}

\section{INTRODUCTION}

Dentin hypersensitivity is one of the most displeasing issues that could be experienced by patients that negatively affect the individual's quality of life. It is characterized by transient, intense, well-localized

Permeability, Forsterite,

adhesive and glass ionomer

1. * Assistant Lecturer of Operative Dentistry, Faculty of Dentistry, Nahda University, Bani-Swef, Egypt. email: sarah.mohsen@nub.edu.eg

2. Professor of Operative Dentistry, Faculty of Dental Medicine for Girls, Al-Azhar University, Cairo, Egypt.

3. Professor of Operative Dentistry, Faculty of Dentistry, Cairo University, Egypt.

4. Associate professor of Operative Dentistry, Faculty of Dentistry, Fayoum University, Egypt 
pain in response to evaporative, tactile, thermal, osmotic or chemical stimuli. Hydrodynamic stimuli lead to inward or outward fluid movement within dentinal tubules that activates mechanoreceptors which is the true physiologic stimulus ${ }^{(1)}$.

Dentin hypersensitivity is treated by either sealing dentinal tubules or making deposits inside them. (2) Occluding or sealing the uncovered dentinal tubules counteracts intratubular fluid shifts which thus give alleviation. There are distinctive procedures for accomplishing this, including precipitation of poorly soluble salts within dentinal tubules or sealing with either polymerizable materials ${ }^{(3)}$ compared to an established single-bottle nanofilled total etch resin adhesive indicated for root desensitization. Methods: Dentin permeability was measured on human crown sections on etched dentin, presenting a model for the exposed tubules typical of root sensitivity, and permitting measurement of the maximum permeability. In the first two groups, the etched dentin was coated with either the RMGI or adhesive, and permeability measured on the coated dentin. In a third group, a smear layer was created on the dentin with sandpaper, then the specimens were coated with the RMGI; permeability was measured on the smeared and coated dentin. Specimens from each group were sectioned and examined via scanning electron microscopy (SEM. Bioactive materials react with body fluids and/or saliva and precipitate hydroxy-carbonate apatite to occlude opened dentinal tubules and treat dentin hypersensitivity ${ }^{(4)}$.

Nano-sized materials with good scattering can enter dentinal tubules of $2-3 \mu \mathrm{m}$ diameters easily; thus, they can be ultimate choice for dentinal tubule occlusion. The greater surface energy and larger surface area fundamentally increase the solvency and reactivity of nanoparticles ${ }^{(5)}$.

Nano Forsterite $\left(\mathrm{Mg}_{2} \mathrm{Sio}_{4}\right)$ is a phase of $\mathrm{MgO}$ $\mathrm{SiO}_{2}$ system that shows high biocompatibility and good mechanical properties ${ }^{(6)}$. Nanoscale Forsterite $\left(\mathrm{Mg}_{2} \mathrm{SiO}_{4}\right)$ is similar to bioactive glass. It forms hydroxy-carbonate apatite layer on the surface of the particles in the biological environment ${ }^{(7)}$.
Similarly, Glass ionomers succeeded in providing instant and long term relief from sensitivity; in addition to their ability to release fluoride ${ }^{(3)} \mathrm{com}$ pared to an established single-bottle nanofilled total etch resin adhesive indicated for root desensitization. Methods: Dentin permeability was measured on human crown sections on etched dentin, presenting a model for the exposed tubules typical of root sensitivity, and permitting measurement of the maximum permeability. In the first two groups, the etched dentin was coated with either the RMGI or adhesive, and permeability measured on the coated dentin. In a third group, a smear layer was created on the dentin with sandpaper, then the specimens were coated with the RMGI; permeability was measured on the smeared and coated dentin. Specimens from each group were sectioned and examined via scanning electron microscopy (SEM. A previous research showed that inclusion of $\mathrm{Mg}_{2} \mathrm{SiO}_{4}$ nanoparticles into glass ionomer cement improved its mechanical properties ${ }^{(8)}$. However, no studies investigated its effect on sealing dentinal tubules for treating dentin hypersensitivity.

The morphological variation of dentinal tubule size and diameter all through the tooth uncovers the dissimilarity between superficial and deep dentin. The tubule density vary widely due to the divergence of dentinal tubules that occurs between the dentino-enamel junction and the pulp ${ }^{(9)}$ although at present there does not appear to be a universally accepted desensitizing agent. Current opinion based on Brännström's Hydrodynamic Theory would suggest that following exposure of the dentin surface (through attrition, abrasion, or erosion.

Therefore, this study was conducted to evaluate and compare the effect of application of Forsterite nanoparticles in different protocols; (Forsterite nanoparticles alone, Forsterite nanoparticles added to an adhesive system and Forsterite nanoparticles incorporated into glass ionomer) on permeability of superficial and deep dentine at different time intervals. 


\section{MATERIALS AND METHODS}

\section{Selection of teeth:}

A total of 30 freshly extracted sound human molars extracted for periodontal reasons were gathered from healthy patients with age range from 30-45 following approval of the ethics committee of Faculty of Dental Medicine for Girls, Al-Azhar University. The patients were informed about the use of their extracted teeth in this study. After cleaning and scaling teeth were stored in distilled water that was changed daily in a refrigerator at $4^{\circ} \mathrm{C}$ to be used within a month after extraction.

\section{Grouping of teeth:}

The 30 molars were used to prepare 60 dentin discs (30 superficial dentin discs and 30 deep dentin discs). Dentin discs were divided into three groups $(\mathrm{n}=20)$ according to the treatment protocol $(\mathrm{P}) ;(\mathrm{P} 1)$ : discs were treated with Forsterite nanoparticles, (P2): discs were treated with Forsterite nanoparticles added to an adhesive system and (P3): discs were treated with Forsterite nanoparticles incorporated into glass ionomer. The main groups were subdivided into two subgroups $(\mathrm{n}=10)$ according to the dentine depth (D); (D1): superficial dentine and (D2): deep dentine. Permeability was measured for each specimen at three time intervals (T); (T1) baseline, (T2) after one week and (T3) after four weeks.

\section{Preparation and characterization of Forster- ite nanoparticles:}

Forsterite nanoparticles were prepared at Nanostreams Company (Science, Technology \& Engineering in 6th of October City, nanostreams@ gmail.com, http://nanostreams.co.uk) by the following method; Forsterite $\left(\mathrm{Mg}_{2} \mathrm{SiO}_{4}\right)$ nanoparticles were prepared by sol-gel method. For making a paste, the Forsterite nanoparticles powder was mixed with the liquid that consists of biocompatible and bio-resorbable natural polymers mainly composed of carboxymethyl cellulose, glucose and polyvinylpyrrolidone together with sodium chloride and magnesium chloride at powder/liquid ratio 2:3 by weight.

The Forsterite nanoparticles were analyzed using $\mathrm{X}$-ray Diffraction (XRD) analysis as an assessment of the development of the Forsterite and particle size. The morphology of the synthesized Forsterite nanoparticles was evaluated by Transmission Electron Microscope (TEM) (model JEM-1230, Joel, Tokyo, Japan) and the particles distribution in the adhesive system and the glass ionomer powder were evaluated using Scanning Electron Microscope (SEM). The samples were coated with a thin layer of Gold $(\mathrm{Au})$ by sputtering and examined by scanning electron microscope model Quanta 250 FEG (Field Emission Gun).

\section{Forsterite nanoparticles addition to the adhesive system:}

Three percent by weight of Forsterite nanoparticles were added to the adhesive liquid, weighed by a precision balance (Nimbus, Adam's equipments, USA). The adhesive solution bottle then it was sonicated in an ultrasonic mixer (Eumax, China) for 30 minutes to attain a miscible solution without any powder precipitate.

\section{Forsterite nanoparticles incorporation into glass ionomer:}

Three percent by weight of Forsterite nanoparticles powder was weighed by a precision balance and added to the glass ionomer powder part.

\section{Preparation of dentine discs:}

The roots of the collected teeth were sectioned; $1 \mathrm{~mm}$ apical to the cementum-enamel junction using a water-cooled diamond saw (BesQual, NY 11373, USA). The pulp tissue was removed carefully with endodontic K-files (M-access, Dentsply, Sirona, Switzerland) and the teeth were washed properly under running water. The occlusal enamel was ground under running water with a grinding wheel (Whip mix, Whip mix corporation, USA) to obtain flat dentin surfaces. Teeth were attached to a pre-fabricated acrylic mold from the root side 
to facilitate their handling and then were sectioned from the flattened occlusal side perpendicular to the long axis of the tooth with an Isomet (Isomet 4000 , Buehler, USA) at a speed of 2,500 rpm and a speed rate of $16.5 \mathrm{~mm} / \mathrm{min}$ under copious amount of water coolant to obtain two discs, superficial and deep (Superficial dentin: Dentin within 0.5-1 mm of dentino-enamel junction and Deep dentin: Dentin within $0.5 \mathrm{~mm}$ of the pulp chamber) ${ }^{(10)}$. The thickness of the disc $1 \mathrm{~mm}( \pm 0.1 \mathrm{~mm})$ was confirmed by a digital caliper. Teeth were detached from the acrylic mold and reattached to it from their occlusal side to expose the root side, the root side was ground till all signs of pulp chamber disappeared and a deep dentin disc of; $1 \mathrm{~mm}( \pm 0.1 \mathrm{~mm})$ thickness was obtained as previously mentioned. Discs were stored in distilled water that was changed daily at $4^{\circ} \mathrm{C}$ till they were treated with the treatment protocols.

\section{Permeability apparatus:}

A fluid transport apparatus was used to test the permeability of the dentin discs. The fluid transport apparatus included 6 Teflon split chamber device (A), each consists of two parts, female and male parts which are attached to each other by screwing action. Two pairs of identical rubber "O" rings with inner space diameter $(0.4 \mathrm{~cm})$ and outer diameter $(1$ $\mathrm{cm})$ sealed the chamber to the discs and controlled the surface area of dentin to be studied. Hydrostatic pressure was applied to one side [(male part (B)] of the chamber from an oxygen pump and was adjusted by an oxygen regulator attached to the apparatus, to induce filtration through the dentin discs across a plastic tube attached to the split chamber (inlet). Thus, water filtration was measured as fluid displacement in a plastic tube connected to the female part $(\mathrm{C})$ of the split chamber with a lumen of $0.7 \mathrm{~cm}$ (outlet) and $35 \mathrm{~cm}$ length ${ }^{(11)}$.

\section{Measurement of baseline fluid filtration:}

Dentin permeability was measured by tracing the displacement of an air bubble in the plastic tube connected to the female part of the split chamber. All the discs where etched on both sides with $37 \%$ phosphoric acid gel for 20 seconds, then rinsed and used to record the baseline permeability of each disc. The discs were placed in the split chamber device between two rubber "O" rings with the pulp sides of the discs facing the pressure pump side (inlet) and the occlusal sides facing the outlet. An air bubble was introduced in the plastic tube connected to the female part of the split chamber. The air bubble location was determined for every specimen. Pressure was then applied through the $\mathrm{O}_{2}$ pump adjusted at $2 \mathrm{~kg} . \mathrm{f} / \mathrm{cm}^{2}$ for 12 hours to the pulpal side of the dentin disc (the inlet of the split-chamber) and the volume of the fluid flowing out from the occlusal side (the outlet) of the split-chamber was measured by the linear displacement of the air bubble in the horizontally positioned rubber tube $(0.7 \mathrm{~cm}$ inside diameter), then the pump was switched off and the air bubble location was determined ${ }^{(12)}$.

Baseline permeability was measured; Permeability was expressed in terms of fluid filtration rate (Jv) and was calculated as follows; Fluid flow (Q) in $\mu 1$ was derived from the linear displacement of the bubble based on the volume of the rubber tube using the following equation ${ }^{(13)}$.

$\mathrm{Q}=$ displacement $\mathrm{x}$ surface area of the rubber tube $(2 \pi r \times h)$.

$(\mathrm{r}=$ inner radius of the tube, $\mathrm{h}=$ length of the tube $)$

Fluid filtration rate $(\mathrm{Jv})$ was calculated by the following equation;

$\mathrm{JV}=\mathrm{Q} / \mathrm{At}$

( $\mathrm{Jv}=$ fluid filtration rate, in $\mu 1 \mathrm{~cm}^{-2} \mathrm{~min}^{-1}, \mathrm{Q}=$ fluid flow in $\mu 1, \mathrm{~A}=$ dentin surface area in $\mathrm{cm}^{-2}$ $\left(\pi r^{2}\right), T=$ time in minutes. $(12$ hours $\times 60=720$ minutes))

\section{Treatment of dentine discs:}

Discs received treatment as assigned by grouping; group P1: discs were treated with Forsterite nanoparticles paste, a graduated insulin syringe was used to standardize the amount applied on each disk, the paste was brushed on the dentine discs using a micro-brush for $20 \mathrm{sec}$ and left on the surface of the disc for 2 min. Group P2: discs were treated 
by Forsterite nanoparticles added to single bond universal at $3 \mathrm{wt} \%$. The adhesive was applied to the dried dentin surface, rubbed by a micro-brush for $20 \mathrm{sec}$, a gentle air stream was directed over the liquid for $5 \mathrm{sec}$ to ensure evaporation of the solvent and light-cured with 3M ESEP light cure (3M ESEP, Elipar ${ }^{\mathrm{TM}}$, Germany) for $15 \mathrm{sec}$ according to the manufacturer's instructions and group P3: discs were treated with Forsterite nanoparticles incorporated into ketac Molar Easymix glass ionomer powder at $3 \mathrm{wt} \%$. The powder was mixed with the liquid according to the manufacturer's instructions the insulin syringe was used to standardize the amount applied on each disk and the material was applied on the surface of the dentin discs using a plastic instrument and left to cure. All discs were then stored in Ringer's solution that was changed daily ${ }^{(14,15)}$. The discs were retested for permeability at one week and four weeks.

\section{Measurement of fluid filtration blockage:}

Each disc was remounted in its specific chamber and fluid filtration was measured the same as in baseline measurements. Permeability reduction values were calculated by subtracting the fluid filtration measurements after 1 week and after 4 weeks from fluid filtration values at baseline.

\section{Environmental Scanning Electron Micro- scope (ESEM) and Energy Dispersive Ana- lytical X-ray (EDAX):}

Two representative samples from each group were chosen to examine the morphological changes that occurred in the dentinal tubules after etching and after application of the different treatment protocols at one week and four weeks. These discs were mounted, occlusal surface facing upward, on an Environmental Scanning Electron Microscope model Quanta 250 FEG (Field Emission Gun) and examined at 2000x. They were photo micrographed, The percentage of open dentinal area was calculated using image analysis by Image J-Fiji program for each disc after etching and after each treatment protocol at one and four weeks interval.

\section{Statistical analysis:}

The mean and standard deviation values were calculated for each group in each test. Data were explored for normality using Kolmogorov-Smirnov and Shapiro-Wilk tests and showed parametric distribution. Three-way ANOVA analysis was used for the effect of different variables on permeability. Paired wise sample t-test was used to compare between two groups in related samples. One-way ANOVA followed by Post Hoc Tukey was used to compare between more than two groups in non-related samples. The significance level was set at $\mathrm{P} \leq$ 0.05 . Statistical analysis was performed with IBM $®$ SPSS ${ }^{\circledR}$ Statistics Version 20 for Windows.

\section{RESULTS}

\section{Results of Characterization of Forsteritenanoparticles:}

Forsterite nanoparticles were analyzed using X-ray Diffraction (XRD) analysis to asses the formation of the Forsterite phase and the size of particles formed and Transmission Electron Microscope to assess particle morphology (TEM). XRD analysis confirmed Forsterite phase formation with Particle size of $\approx 30 \mathrm{~nm}$. TEM showed Narrow particle size distribution with slight agglomeration. Scanning electron microscope micrographs showed almost even distribution of Forsterite nanoparticles in the adhesive system and the glass ionomer powder.

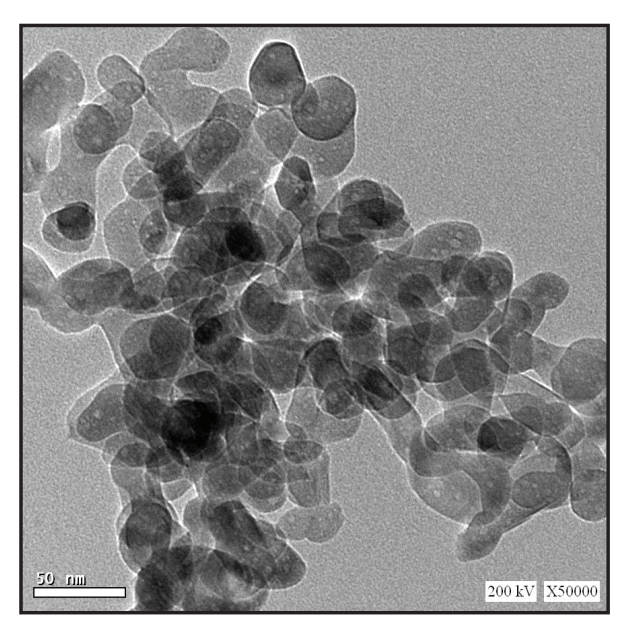

Figure (1) TEM of Forsterite nanoparticles 


\section{Dentin permeability reduction results:}

Three-way ANOVA analysis for the effect of different variables on dentin permeability reduction showed that the treatment protocols had a statistically significant effect at F-value 42.794 and $\mathrm{P}$-value $<0.001$. Dentin depth also had a statistically significant effect on mean dentin permeability reduction at F-value 90.447 and P-value $<0.001$ and time had a statistically significant effect on mean dentin permeability reduction at F-value 174.024 and $\mathrm{P}$-value $<0.001$. While the interaction between the three variables had no statistically significant effect on mean dentin permeability reduction at F-value 0.288 and $\mathrm{P}$-value 0.751 .

Effect of treatment protocols on dentin permeability reduction

At 1 week (T2) and 4 weeks (T3), a statistically significant difference was found between tested groups where $(p=0.004)$ and $(p<0.001)$.

For superficial dentin discs, at 1 week (T2), Forsterite nanoparticles added to an adhesive system (P2) had the highest mean value $(0.104 \pm 0.011)$ in comparison to Forsterite nanoparticles (P1) (0.084 $\pm 0.011)$ and Forsterite nanoparticles incorporated in Glass Ionomer (P3) (0.074 \pm 0.011$)$. However, no statistically significant difference was found between Forsterite nanoparticles (P1) and Forsterite nanoparticles incorporated in Glass Ionomer (P3) groups. At 4 weeks (T3), Forsterite nanoparticles (P1) had the highest mean value $(0.200 \pm 0.158)$ in comparison to Forsterite nanoparticles added to an adhesive system (P2) $(0.136 \pm 0.024)$ and Forsterite nanoparticles incorporated in Glass Ionomer (P3) $(0.130 \pm 0.019)$. Whereas, no statistically significant difference was found between Forsterite nanoparticles added to an adhesive system (P2) and Forsterite nanoparticles incorporated in Glass Ionomer (P3) groups as shown in table (1).

For deep dentin discs, at 1 week (T2) and 4 weeks (T3), a statistically significant difference was found between tested groups where $(p=0.001)$ and $(p<0.001)$. at 1 week (T2), the highest mean value was found in Forsterite nanoparticles added to an adhesive system (P2) $(0.170 \pm 0.016)$ followed by Forsterite nanoparticles (P1) $(0.134 \pm 0.021)$, while, Forsterite nanoparticles incorporated in Glass Ionomer (P3) showed the lowest value at $(0.114$ $\pm 0.015)$. At 4 weeks (T3), the highest mean value was found in Forsterite nanoparticles (P1) (0.230 $\pm 0.016)$ in comparison to Forsterite nanoparticles added to an adhesive system (P2) $(0.188 \pm 0.018)$ and Forsterite nanoparticles incorporated in Glass Ionomer (P3) $(0.140 \pm 0.019)$. While no statistically significant difference was found between Forsterite nanoparticles added to an adhesive system (P2) and Forsterite nanoparticles incorporated in Glass Ionomer (P3) groups as shown in table (1).

\section{Effect of dentin depth on dentin permeability reduction:}

For Forsterite nanoparticles (P1), a statistically significant difference was found between superficial dentin disc (D1) and deep dentin disc (D2) at 1 week (T2) and 4 weeks (T3) where $(p=0.001)$ and $(p=0.017)$ respectively. The higher mean value was found in superficial dentin disc (D2) at 4 week $(0.230 \pm 0.016)$, the lowest mean value was found in deep dentin (D1) at 1 week $(0.084 \pm 0.021)$ as shown in table (2).

For Forsterite nanoparticles added to an adhesive system (P2) at 1 week (T2), a statistically significant difference was found between superficial dentin disc (D1) and deep dentin disc (D2) where $(p=0.002)$. The superficial dentin disc (D2) showed higher values $(0.114 \pm 0.015)$ in comparison to the deep dentin disc (D1) $(0.074 \pm 0.011)$. Moreover, at 4 weeks (T3), no statistically significant difference was found between superficial dentin disc (D1) and deep dentin disc (D2) where ( $p=0.423)$ as shown in table (2). 
Table (1): The mean and standard deviation (SD) values of dentin permeability reduction of different treatment protocols at different time periods for superficial and deep dentin discs:

\begin{tabular}{|c|c|c|c|c|c|c|c|c|}
\hline \multirow{3}{*}{ Variables } & \multicolumn{4}{|c|}{ Superficial dentin discs (D1) } & \multicolumn{4}{|c|}{ Deep dentin discs (D2) } \\
\hline & \multicolumn{2}{|c|}{1 week (T2) } & \multicolumn{2}{|c|}{4 weeks (T3) } & \multicolumn{2}{|c|}{1 week (T2) } & \multicolumn{2}{|c|}{4 weeks (T3) } \\
\hline & Mean & SD & Mean & SD & Mean & SD & Mean & SD \\
\hline Forsterite nanoparticles (P1) & $0.084^{b}$ & 0.011 & $0.200^{\mathrm{a}}$ & 0.0158 & $0.134^{\mathrm{b}}$ & 0.021 & $0.230^{\mathrm{a}}$ & 0.016 \\
\hline $\begin{array}{l}\text { Forsterite nanoparticles added to an adhe- } \\
\text { sive system (P2) }\end{array}$ & $0.104^{\mathrm{a}}$ & 0.011 & $0.136^{\mathrm{b}}$ & 0.024 & $0.170^{\mathrm{a}}$ & 0.016 & $0.188^{\mathrm{b}}$ & 0.018 \\
\hline $\begin{array}{l}\text { Forsterite nanoparticles incorporated in } \\
\text { Glass Ionomer (P3) }\end{array}$ & $0.074^{\mathrm{b}}$ & 0.011 & $0.130^{\mathrm{b}}$ & 0.019 & $0.114^{\mathrm{c}}$ & 0.015 & $0.140^{\mathrm{b}}$ & 0.019 \\
\hline p-value & \multicolumn{2}{|c|}{$0.004 *$} & \multicolumn{2}{|c|}{$<0.001 *$} & \multicolumn{2}{|c|}{$0.001 *$} & \multicolumn{2}{|c|}{$<0.001^{*}$} \\
\hline
\end{tabular}

Superscripts with different small letters indicate statistically significance difference

*; significant $(p \leq 0.05) \quad n s ;$ non-significant $(p>0.05)$

Table (2): The mean and standard deviation (SD) values of dentin permeability reduction in superficial and deep dentin discs at different time periods in the forestrite nanoparticles group (P1), Forsterite nanoparticles added to an adhesive system (P2) and Forsterite nanoparticles incorporated into glass ionomer (P3):

\begin{tabular}{|c|c|c|c|c|c|c|c|c|c|c|c|c|}
\hline \multirow{3}{*}{ Variables } & \multicolumn{4}{|c|}{ Forsterite nanoparticle (P1) } & \multicolumn{4}{|c|}{$\begin{array}{c}\text { Forsterite nanoparticles added to } \\
\text { an adhesive system (P2) }\end{array}$} & \multicolumn{4}{|c|}{$\begin{array}{l}\text { Forsterite nanoparticles incorpo- } \\
\text { rated into glass ionomer }(\mathbf{P 3})\end{array}$} \\
\hline & \multicolumn{2}{|c|}{1 week (T2) } & \multicolumn{2}{|c|}{4 weeks (T3) } & \multicolumn{2}{|c|}{1 week (T2) } & \multicolumn{2}{|c|}{4 weeks (T3) } & \multicolumn{2}{|c|}{1 week (T2) } & \multicolumn{2}{|c|}{4 weeks (T3) } \\
\hline & Mean & SD & Mean & SD & Mean & SD & Mean & SD & Mean & SD & Mean & SD \\
\hline Superficial dentin(D1) & $0.134^{\mathrm{a}}$ & 0.011 & $0.230^{\mathrm{a}}$ & 0.016 & $0.114^{\mathrm{a}}$ & 0.015 & $0.140^{\mathrm{a}}$ & 0.019 & $0.170^{\mathrm{a}}$ & 0.016 & $0.188^{\mathrm{a}}$ & 0.018 \\
\hline Deep dentin(D2) & $0.084^{\mathrm{b}}$ & 0.021 & $0.200^{\mathrm{b}}$ & 0.016 & $0.074^{\mathrm{b}}$ & 0.011 & $0.130^{\mathrm{a}}$ & 0.019 & $0.104^{\mathrm{b}}$ & 0.011 & $0.136^{\mathrm{b}}$ & 0.024 \\
\hline$p$-value & \multicolumn{2}{|c|}{$0.001 *$} & \multicolumn{2}{|c|}{$0.017 *$} & \multicolumn{2}{|c|}{$0.002 *$} & \multicolumn{2}{|c|}{$0.423 \mathrm{~ns}$} & \multicolumn{2}{|c|}{$<0.001 *$} & \multicolumn{2}{|c|}{$0.005^{*}$} \\
\hline
\end{tabular}

Superscripts with different small letters indicate statistically significance difference. $\quad * ; \quad$ significant $\quad(p \leq 0.05)$ $n s ;$ non-significant ( $p>0.05)$

For Forsterite nanoparticles incorporated into glass ionomer (P3), a statistically significant difference was found between superficial dentin disc (D1) and deep dentin disc (D2) at 1 week (T2) and 4 weeks (T3) where $(p<0.001)$ and $(p=0.005)$ respectively. The highest mean value was found in superficial dentin disc (D2) at 4 week $(0.188 \pm$ 0.018 ), the lowest mean value was found in deep dentin disc (D1) 1 week $(0.104 \pm 0.011)$ as shown in table (2).

\section{Effect of time on dentin permeability reduction in each group:}

For superficial dentin discs, in Forsterite nanoparticles (P1), Forsterite nanoparticles added to an adhesive system (P2) and Forsterite nanoparticles incorporated in Glass Ionomer (P3) a statistically significant difference was found between 1 week (T2) and 4 weeks (T3) where $(p<0.001)$, $(p=0.012)$ and $(p=0.005)$. A higher mean value was found in 4 weeks (T3), the lower mean value was found in 1 week as shown in table (3). 
Table (3): The mean and standard deviation $(S D)$ values of dentin permeability reduction at different time periods of different treatment protocols for superficial and deep dentin discs:

\begin{tabular}{|c|c|c|c|c|c|c|c|c|c|c|c|c|}
\hline \multirow{3}{*}{ Variables } & \multicolumn{6}{|c|}{ Superficial dentin disc (D1) } & \multicolumn{6}{|c|}{ Deep dentin disc (D2) } \\
\hline & \multicolumn{2}{|c|}{$\begin{array}{c}\text { Forsterite } \\
\text { nanoparticle } \\
\text { (P1) }\end{array}$} & \multicolumn{2}{|c|}{$\begin{array}{c}\text { Forsterite } \\
\text { nanoparticle } \\
\text { added to an ad- } \\
\text { hesive system } \\
\text { (P2) }\end{array}$} & \multicolumn{2}{|c|}{$\begin{array}{c}\text { Forsterite } \\
\text { nanoparticle } \\
\text { incorporated in } \\
\text { Glass Ionomer } \\
\text { (P3) }\end{array}$} & \multicolumn{2}{|c|}{$\begin{array}{c}\text { Forsterite } \\
\text { nanoparticle } \\
\text { (P1) }\end{array}$} & \multicolumn{2}{|c|}{$\begin{array}{c}\text { Forsterite } \\
\text { nanoparticle } \\
\text { added to an ad- } \\
\text { hesive system } \\
\text { (P2) }\end{array}$} & \multicolumn{2}{|c|}{$\begin{array}{c}\text { Forsterite } \\
\text { nanoparticle } \\
\text { incorporated in } \\
\text { Glass Ionomer } \\
\text { (P3) }\end{array}$} \\
\hline & Mean & SD & Mean & SD & Mean & SD & Mean & SD & Mean & SD & Mean & SD \\
\hline 1 week (T2) & $0.084^{b}$ & 0.011 & $0.104^{b}$ & 0.011 & $0.074^{\mathrm{b}}$ & 0.011 & $0.134^{\mathrm{b}}$ & 0.021 & $0.170^{b}$ & 0.016 & $0.114^{b}$ & 0.015 \\
\hline 4 weeks (T3) & $0.200^{\mathrm{a}}$ & 0.016 & $0.136^{\mathrm{a}}$ & 0.024 & $0.130^{\mathrm{a}}$ & 0.019 & $0.230^{\mathrm{a}}$ & 0.016 & $0.188^{\mathrm{a}}$ & 0.018 & $0.140^{\mathrm{a}}$ & 0.019 \\
\hline p-value & \multicolumn{2}{|c|}{$<0.001 *$} & \multicolumn{2}{|c|}{$0.012 *$} & \multicolumn{2}{|c|}{$0.005^{*}$} & \multicolumn{2}{|c|}{$0.002 *$} & \multicolumn{2}{|c|}{$0.001 *$} & \multicolumn{2}{|c|}{$0.007 *$} \\
\hline
\end{tabular}

Superscripts with different small letters indicate statistically significance difference within the same column. *; significant $(p \leq 0.05) \quad n s ;$ non-significant $(p>0.05)$

For deep dentin discs, in Forsterite nanoparticles (P1), Forsterite nanoparticles added to an adhesive system (P2) and Forsterite nanoparticles incorporated in Glass Ionomer (P3) a statistically significant difference was found between 1 week (T2) and 4 weeks (T3) where ( $\mathrm{p}=0.002),(\mathrm{p}=0.001)$ and $(\mathrm{p}=0.007)$. A higher mean value was found in 4 weeks (T3), the lower mean value was found in 1 week as shown in table (3).

\section{Environmental Scanning Electron Microscope observation:}

ESEM observation of both superficial and deep etched dentin discs of all groups showed opened and patent dentinal tubules, superficial dentin disc showed $23 \%, 27.2 \%$ and $22.5 \%$ of opened dentinal area after etching (fig:1a, fig: $2 \mathrm{a}$ and fig: $3 \mathrm{a}$ ). While deep dentin discs showed 28\%, 29.5\% and $28.9 \%$ of open dentinal area. (fig: 1d, fig: $2 \mathrm{~d}$ and fig: $3 \mathrm{~d}$ )

After 1 week, ESEM scan of superficial and deep dentin disc showed closure of dentinal tubule opening. Superficial dentin disc showed $12.34 \%$ reduction in open dentinal area. While, Deep dentin showed $19.6 \%$ reduction in open dentinal area in Forsterite nanoparticles [fig: $1 \mathrm{~b}$ and 1e]. In
Forsterite nanoparticles added to an adhesive system, superficial dentin at one week of treatment showed $26.22 \%$ reduction in open dentinal area (fig: $2 b)$. Deep dentin at one week of treatment showed $27.51 \%$ reduction in open dentinal area (fig: $2 \mathrm{e}$ ). As for Forsterite nanoparticles incorporated into glass ionomer, superficial dentin at one week of treatment showed $18.5 \%$ reduction in open dentinal area (fig: $3 b)$. Deep dentin at one week of treatment showed $24.5 \%$ reduction in open dentinal area. (fig: $3 \mathrm{e}$ )

After 4 weeks, superficial dentin disc showed $22.86 \%$ reduction in open dentinal area (fig: 1c). Deep dentin disc after four weeks of treatment showed $27.6 \%$ reduction in open dentinal area in Forsterite nanoparticles (fig: 1f). Superficial dentin disc showed $27.02 \%$ reduction in open dentinal area and deep dentin disc showed $29.33 \%$ reduction in open dentinal area in Forsterite nanoparticles added to an adhesive system (fig: $2 \mathrm{c}$ and $2 \mathrm{f}$ ). As for Forsterite nanoparticles incorporated into glass ionomer, superficial dentin disc showed $22.34 \%$ reduction in open dentinal area. Deep dentin disc showed $27.68 \%$ reduction in open dentinal area. (fig: $3 \mathrm{c}$ and $3 \mathrm{f}$ ) 


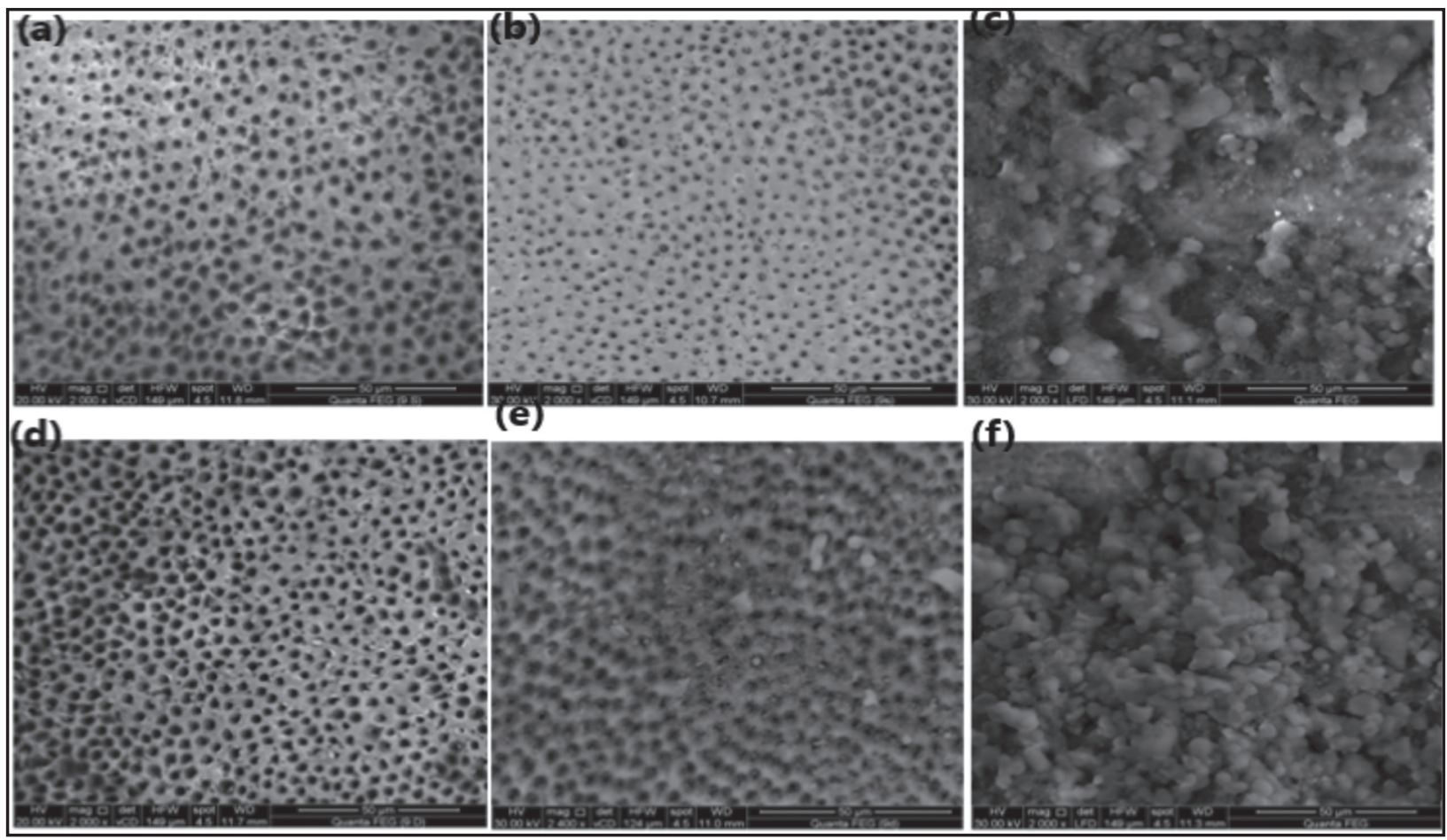

Figure (2) ESEM micrographs of superficial dentin disc of Forsterite nanoparticles group after etching (a), after 1 week of treatment (b) and after 4 weeks of treatment (c) and deep dentin disk after etching (d), after 1 week of treatment (e) and after 4 weeks of treatment (f) at 2000x
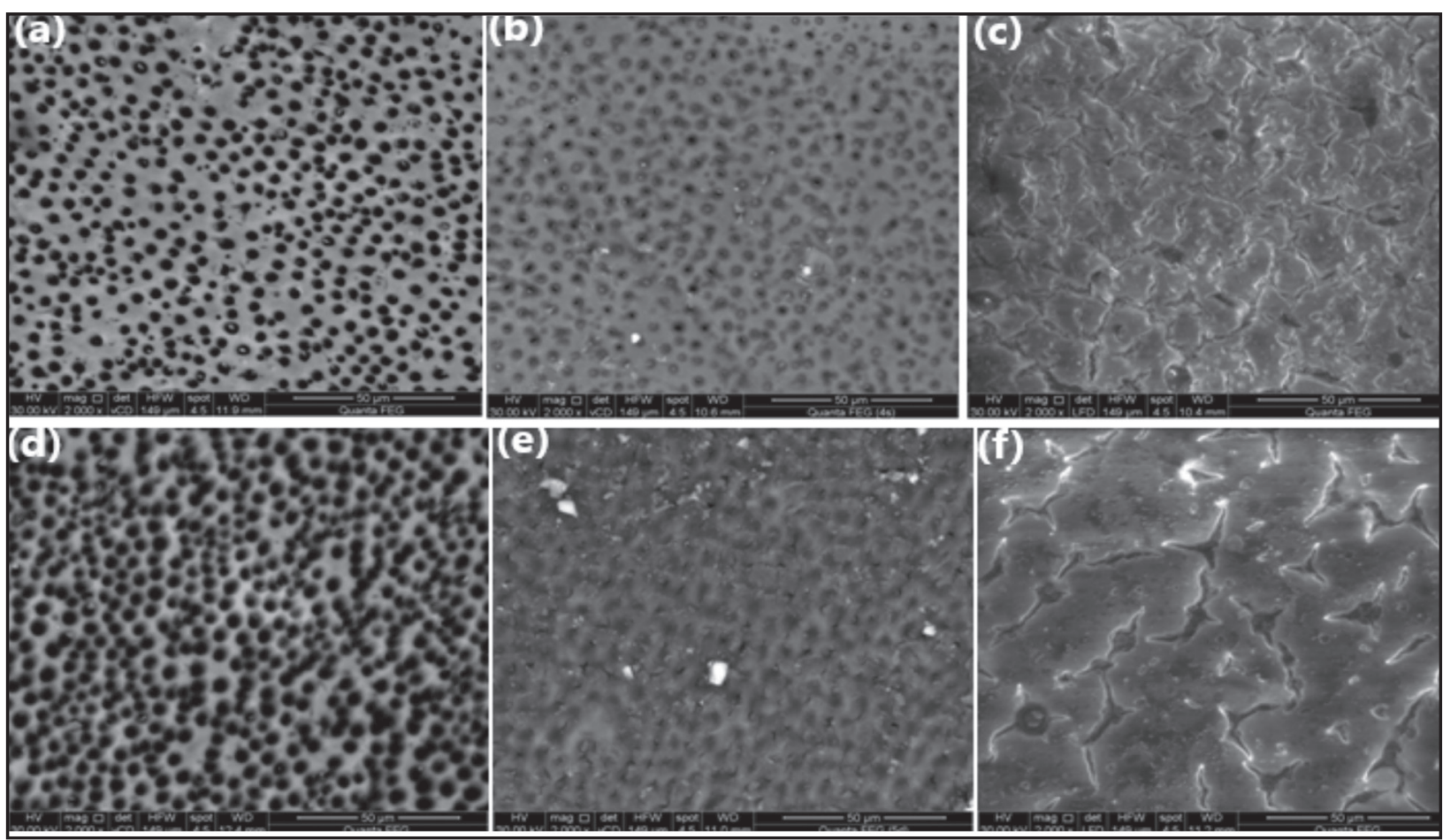

Figure (3) ESEM micrographs of superficial dentin disc of Forsterite nanoparticles added to an adhesive system group after etching (a), after 1 week of treatment (b) and after 4 weeks of treatment (c) and deep dentin disk after etching(d), after 1 week of treatment (e) and after 4 weeks of treatment (f) at 2000x 


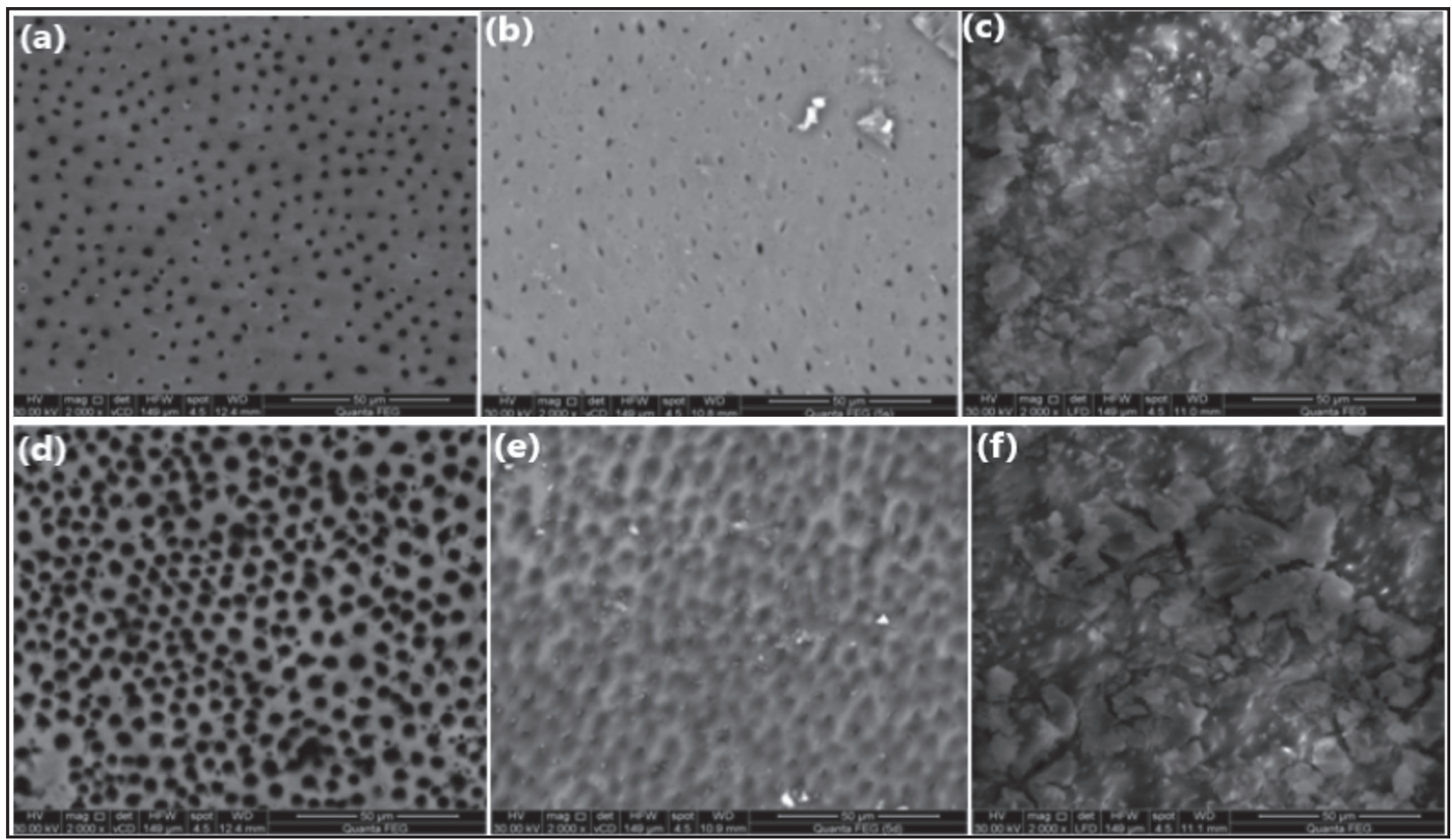

Figure (4) ESEM micrographs of superficial dentin disc of Forsterite nanoparticles incorporated into glass ionomer group after etching (a), after 1 week of treatment (b) and after 4 weeks of treatment (c) and deep dentin disk after etching (d), after 1 week of treatment (e) and after 4 weeks of treatment (f) at 2000x

\section{Energy Dispersive X-ray Analysis (EDAX):}

EDAX of both etched superficial and deep dentin discs showed peaks of calcium and phosphate which are the main components of the tooth structure. In Forsterite nanoparticles after 1 week EDAX of superficial and deep dentin discs showed traces of magnesium, Oxygen and silicate corresponding to application of Forsterite $\left(\mathrm{Mg}_{2} \mathrm{SiO}_{4}\right)$ and after 4 weeks, EDAX of both superficial and deep dentin discs showed elevated silica and magnesium peaks and decreased calcium and phosphate peaks.

In Forsterite nanoparticles added to an adhesive system, after 1 week, EDAX of both groups showed peaks of magnesium, silica and oxygen which are the main components of Forsterite added to the adhesive system and after 4 weeks, both groups showed elevated peaks of silica more pronounced in deep dentin disc. As for Forsterite nanoparticles incorporated into glass ionomer, EDAX of both groups showed peaks of aluminum and sodium from glass ionomer and peaks of oxygen and silica from Forsterite incorporated into the glass ionomer. While after 4 weeks, the two groups showed elevated peaks of silica.

\section{DISCUSSION}

The dentin flow movement toward the pulp or flow in the opposite direction when a stimulus is exerted to dentin causes mechanical stimulation of the nerve fibers inside the tubule or at the interface of pulp and dentin, transmitted as an excruciating sensation ${ }^{(16)}$. The Hagen- Poiseuille equation states the proportionality between dentin hypersensitivity and both the forth power of tubular radius and to the pressure differences at tubule ends ${ }^{(17)}$. Thus, narrowing of dentinal tubules or blocking them could effectively reduce dentin hypersensitivity by decreasing dentin permeability ${ }^{(18)}$.

A study was conducted to investigate the relationship between dentin permeability and dentin depth and proved that as dentin depth increase permeability increases. Tubule diameter and number 
increase towards the pulp chamber which explains this relationship. Hence, in this study two different dentin depths; superficial and deep dentin were included $^{(19)}$.

Bioactive glasses are biocompatible materials traditionally used for osteogenesis but recently because of their resemblance with bone and dentin, they are used in the field of dentistry ${ }^{(20)}$. They induce precipitation of calcium phosphate complexes and in appropriate particle size BGs has the potential to occlude dentinal tubules ${ }^{(21,22)}$, as well as promoting dentine remineralization ${ }^{(23-26)}$. Extensive research on using bioactive glasses in the treatment of dentin hypersensitivity was carried out ${ }^{(27-30)}$.

In the current study Forsterite $\left(\mathrm{Mg}_{2} \mathrm{SiO}_{4}\right)$ was used, an important material in the magnesia-silica system which showed significantly improved properties compared with hydroxyapatite ceramics ${ }^{(31)}$. Similar to bioactive glass, Forsterite contains essential ions that are released leading to precipitation of hydroxi-carbonate apatite layer ${ }^{(32)}$.

According to a previous research on simulated body fluids and synthetic saliva, some silica compounds could induce apatite formation ${ }^{(5)}$. Silica nanoparticles promoted infiltration and remineralization of dentin ${ }^{(33)}$. The diminished molecular size and huge surface to volume area exhibited by nanosized silica particles improved its solubility due to increased area exposed to dissolution ${ }^{(26)}$. When compared with micron-sized particles nanosized silica posses improved remineralization potential and ability to be impeded within the dentinal tubules ${ }^{(34)}$. Therefore, in the current study Forsterite nanopowder with particle size $\sim 30 \mathrm{~nm}$ was used.

Moreover, Forsterite nanopowder is bioactive and possesses apatite-forming ability. Because the lumen of the dentinal tubules is a calcium-rich environment, there is a strong possibility that HA particles could be deposited inside the tubules ${ }^{(32,35)}$. This would result in re-mineralization process that promotes a strong and permanent adhesion between the biomaterial and the tooth ${ }^{(36)}$.
Similarly, resin infiltration was suggested to occlude the dentinal tubules, since then, the use of polymer resins to seal dentinal tubules is gaining recognition ${ }^{(13)}$. It was also postulated that polymeric resin suspensions block the dentinal tubules by coagulation of dentine fluid proteins within the tubules thereby counteracting the hydrodynamic mechanism of dentin hypersensitivity ${ }^{(37)}$. Adhesive systems containing HEMA as a hydrophilic primer are common in many modern dentin bonding systems and allow an efficient dentin bonding, they are indicated for treating dentin sensitivity ${ }^{(38)}$. A previous study revealed reduction in dentin permeability using dental adhesive with a range of $16 \%$ to $98 \%{ }^{(39,40)}$. Based on this fact, in this study, Forsterite nano-powder was added to an adhesive system to study their combined effect on dentin permeability.

Restorative techniques such as glass ionomer are used when esthetic and functional re-establishment of lesions is required besides control of hypersensitivity ${ }^{(38)}$, as permeability reduction ranging from $73.8 \%$ to $93.1 \%$ was reported for glass ionomer. Chemical bonding is considered a significant factor in the sealing ability of glass ionomer materials ${ }^{(3)}$ compared to an established single-bottle nanofilled total etch resin adhesive indicated for root desensitization. Methods: Dentin permeability was measured on human crown sections on etched dentin, presenting a model for the exposed tubules typical of root sensitivity, and permitting measurement of the maximum permeability. In the first two groups, the etched dentin was coated with either the RMGI or adhesive, and permeability measured on the coated dentin. In a third group, a smear layer was created on the dentin with sandpaper, then the specimens were coated with the RMGI; permeability was measured on the smeared and coated dentin. Specimens from each group were sectioned and examined via scanning electron microscopy (SEM. Hence, to study the combined effect of glass ionomer and Forsterite on dentin permeability, Forsterite nanopowder was incorporated into the glass ionomer powder by $3 \mathrm{wt} \%{ }^{(8,41)}$. 
The evaluation of apatite and /or mineral complex depositions in scaffolds, when immersed in simulated body fluids, is a commonly accepted method to establish the bioactivity of a constituent to remineralization ${ }^{(42)}$. Ringer's solution was used based on previous studies ${ }^{(14,15)}$.

In-vitro studies on dentin hypersensitivity embrace the use of dentin discs to study dentinal morphology and its interaction with different materials. To study dentin hypersensitivity various methods have been used, utilizing devices to measure dentin permeability or SEM analysis to elaborate dentin reactivity with desensitizing agents ${ }^{(43)}$. Studies comparing desensitizing agents in-vitro have evaluated dentine permeability by measuring fluid movement using a fluid filtration system for hydraulic conductance measurement ${ }^{(44,45-47)}$. It analyses the ability of potential desensitizing agents to reduce fluid flow due to tubule occlusion. Standardization of the uncovered dentin area for dentin permeability assessment was accomplished by using O-rings ${ }^{(48)}$. Thereby, the same area of the same specimen was evaluated each time to ensure reproducibility of the measurements.

The removal of the smear layer was important for imitation of the hypersensitive dentin with patent dentin tubules. It was reported that $86 \%$ of the total resistance to the movement of fluids was due to the smear layer ${ }^{(3)}$ compared to an established singlebottle nanofilled total etch resin adhesive indicated for root desensitization. Methods: Dentin permeability was measured on human crown sections on etched dentin, presenting a model for the exposed tubules typical of root sensitivity, and permitting measurement of the maximum permeability. In the first two groups, the etched dentin was coated with either the RMGI or adhesive, and permeability measured on the coated dentin. In a third group, a smear layer was created on the dentin with sandpaper, then the specimens were coated with the RMGI; permeability was measured on the smeared and coated dentin. Specimens from each group were sectioned and examined via scanning electron microscopy (SEM.
Thus dentin discs were etched on both sides to remove the smear layer to eliminate any interference in the results permeability reduction ${ }^{(13)}$.

For both superficial and deep dentin discs after 1 week, Forsterite nanoparticles added to an adhesive system had the highest mean value in comparison to Forsterite nanoparticles and Forsterite nanoparticles incorporated in glass ionomer. This could be explained by the blockage of the tubules instantly with the application of the adhesive material that has the ability to plug the open dentinal tubules as application of Self Etch adhesives topically effectively reduced dentin permeability by sealing the dentin surface with an acid-resistant hybrid layer and by occluding the tubule orifices with resin tags as reported by some studies ${ }^{(48,49)}$. This finding was in accordance with several previous studies $(50,13,48)$ and in contradiction with studies that used different methodology for surface treatment of the dentin discs by creating a smear layer with a 600 grit silicon carbide paper and applied a drop of $10 \% \mathrm{NaOCl}$ for 60 seconds before application of treatment materials which might have affected the patency of the tubules ${ }^{(51)}$.

After 4 weeks, Forsterite nanoparticles had the highest mean value in comparison to Forsterite nanoparticles added to an adhesive system and Forsterite nanoparticles incorporated in glass ionomer. This is mostly due to the ability of Forsterite nanoparticles to form mineral deposits that blocked the dentinal tubules when stored in the ringer's solution for four weeks. This was previously proposed by a study by Kharaziha and Fathi, $2009^{(32)}$, that Forsterite nanopowder has an apatite forming ability when immersed in a simulated body fluid. Magnesium ions in the powder first exchange with $\mathrm{H}^{+}$in the solution forming silanol $\left(\mathrm{Si}-\mathrm{OH}^{-}\right)$in the surface layer, a PH increase and finally the production of a surface with the functional group with a negative charge $\left(\mathrm{Si}^{-} \mathrm{OH}^{-}\right)$. The calcium ions in the simulated body fluid is drawn to the boundary between the powder and the solution initially that results in calcium accumulating in the silica-rich 
layer and decrease of phosphorus concentration in the solution. Presence of enough calcium and phosphorus on the Forsterite nanopowder causes formation of calcium-phosphate layer on the surface of the nanopowder.

For both superficial and deep dentin discs after 1 week and after 4 weeks, glass ionomer had the lowest mean values, though it was expected that glass ionomer would exhibit better permeability reduction as glass ionomer forms chemical bonds with dentin via ionic and polar bonds and release and exchange of fluoride is facilitated by close molecular contact $^{(52)}$. This may be explained by the fact that acid etching of dentin discs removed calcium ions which are essential for bonding glass ionomer with dentin surface, so this might have decreased its ability to bond to tooth structure and seal the dentinal tubules.

For Forsterite nanoparticles and Forsterite nanoparticles incorporated into glass ionomer, a statistically significant difference was found between superficial dentin disc and deep dentin discs after 1 week and 4 weeks, where higher mean value was found in superficial dentin. For Forsterite nanoparticles added to an adhesive system after 1 week, superficial dentin exhibited statistically significantly higher mean values than deep dentin.

This could be attributed to the fact that deep dentin has dentinal tubules that are more in number per unit area and wider in diameter. This makes deep dentin more permeable ${ }^{(53)}$. The number of dentinal tubules is enhanced in a corono-apical direction per unit area due to the divergence of tubules as they approach the pulp.

As for Forsterite nanoparticles added to an adhesive system after 4 weeks, no statistically significant difference was found between superficial and deep dentin discs, this might be due to the blocking effect of the Forsterite nanoparticles added to the adhesive system on both discs and it is confirmed by the SEM micrographs
For both superficial and deep dentin discs, a statistically significant difference was found between all tested groups after 1 week and 4 weeks of treatment. The highest mean value was recorded after 4 weeks in comparison to 1 week time interval. As for the Forsterite nanoparticles group, this might be attributed to the fact that after one week, there was not enough time for the Forsterite to show any signs of remineralization and block the dentinal tubules. It was reported that the enhancement of the soaking time from 7 to 14 and 21 days enhances the condensation of the nuclei of apatite joining each other to form a layer of the hydroxyapatite over the whole surface $^{(8)}$.

Also for Forsterite nanoparticles incorporated in Glass Ionomer, the results could be explained by the fact that the Forsterite nanoparticles incorporated in Glass Ionomer had little effect after one week of the treatment but after four weeks mineral deposits were formed that blocked the dentinal tubules and decreased permeability. Sayeddan et al $2013^{(8)}$, also stated that the rate of nucleation of apatite on the surface of Forsterite -glass ionomer nanocomposite was enhanced.

Two representative discs of each treatment group were scanned with ESEM. Observations for all etched dentin discs both superficial and deep showed dentinal tubules open and patent. There was an opened dentinal area of 23\%,27.2\% and 22.5\% for superficial dentin discs in Forsterite nanoparticles, Forsterite nanoparticles added to an adhesive system and Forsterite nanoparticles incorporated into glass ionomer, respectively and an open dentinal area of $28 \%, 29.5 \%$ and $28.9 \%$ in deep dentin discs.

After 1 week of treatment, the discs treated with Forsterite nanoparticles, superficial and deep showed partial closure of dentinal tubule openings with white deposits with $10.66 \%$ opened dentinal tubules area and $12.34 \%$ reduction in dentinal tubule area for superficial disc and $8.4 \%$ of open dentinal area and $19.6 \%$ reduction in open dentinal 
area for deep dentin disc. After 4 weeks, superficial and deep dentin disc showed almost complete closure of dentinal tubule openings with deposits with $0.14 \%$ of open dentinal area and $22.86 \%$ reduction in open dentinal area for superficial disc and $\% 0.4$ of open dentinal area and $27.6 \%$ reduction in open dentinal area for deep dentin disc.

After 1 week, discs treated with Forsterite nanoparticles with an adhesive system, superficial dentin disc showed partial closure of some dentinal tubule openings and closure of other dentinal tubule openings with $0.98 \%$ of open dentinal area and $26.22 \%$ reduction in open dentinal area. While, deep dentin disc showed almost complete closure of dentinal tubule openings with $1.99 \%$ of open dentinal area and $27.51 \%$ reduction in open dentinal area. After 4 weeks, superficial and deep dentin discs showed completely closed dentinal tubule openings with $0.18 \%$ of open dentinal area and $27.02 \%$ reduction in open dentinal area for superficial dentin disc and with $0.17 \%$ of open dentinal area and $29.33 \%$ reduction in open dentinal area for deep dentin disc.

After 1 week, discs treated with Forsterite nanoparticles incorporated in glass ionomer, superficial and deep dentin discs showed partial closure of dentinal tubule openings with white deposits with $4 \%$ of open dentinal area and $18.5 \%$ reduction in open dentinal area for superficial dentin disc and $4.5 \%$ of open dentinal area and $24.5 \%$ reduction in open dentinal area for deep dentin disc. After 4 weeks, superficial and deep dentin disc showed areas with almost completely closed dentinal tubules with deposits and other areas with partially closed dentinal tubules with no deposits with $0.16 \%$ of open dentinal area and $22.34 \%$ reduction in open dentinal area for superficial dentin disc and $\% 1.22$ of open dentinal area and $27.68 \%$ reduction in open dentinal area for deep dentin disc.

ESEM findings of this study support the results of the permeability showing a greater opened dentinal area in deep dentin discs than superficial dentin discs and decrease in open dentinal area with time. The micrographs also showed that Forsterite added to adhesive system exhibited the highest percentage of closure of the opened dentinal tubules in both superficial and deep dentin discs after 1 week which supports the dentin permeability results.

On the other hand, After 4 weeks, Forsterite added to adhesive system showed the highest reduction in percentage of opened dentinal tubules area in superficial and deep dentin discs which is in contradiction with the dentin permeability results. This could be due to the fact that ESEM only shows the surface of the dentine disc which was properly sealed with the adhesive, but it could not show whether the Forsterite nanoparticles penetrated the dentinal tubules or not by forming minerals within the tubules which might result in the blockage

In all the treatment groups, EDAX analysis showed gradual decrease in calcium and phosphate after one week then after four weeks and gradual increase in silica. This might be due to complete coverage of the tooth surface with the materials which concealed the calcium and phosphate of the tooth itself. The increase in silica could be due to the formation of a silica rich layer on the surface of the material in the process of apatite formation ${ }^{(36)}$.

The decrease in calcium may also be due to the formation of a calcium deficient hydroxyl-apatite layer as reported by Ma et al, $2017^{(18)}$ who found that the $\mathrm{Ca} / \mathrm{P}$ ratio in the experimental groups was lower than that of the control group and considered that the calcium and phosphate precipitates induced by the bioactive glass used in the study, combined with dentin surface well.

\section{CONCLUSION}

Within the limitations of the present study, the following conclusions could be drawn:

1. The use of Forsterite nanoparticles in different protocols is effective in reducing dentin permeability in superficial and deep dentin, though 
the addition of Forsterite nanoparticles to glass ionomer was the least effective.

2. Superior reduction of dentin permeability could be achieved after one week of using Forsterite nanoparticles added to the adhesive system and four weeks after using Forsterite nanoparticles alone.

\section{REFERENCES}

1. Pashley DH, Tay FR. Dentin Hypersensitivity: Current State of The Art and Science. Dentine Hypersensitivity Consensus Monograph 2008; 4: 1-11.

2. Davari AR, Ataei E,Assarzadeh H.Dentin Hypersensitivity: Etiology, Diagnosis and Treatment; A Literature Review. J Dent (Shiraz) 2013; 14: 136-45.

3. Rusin RP, Agee K, Suhcko M, Pashley DH. Effect of a New Desensitizing Material on Human Dentine Permeability. Dent Mater J 2010; 26: 600-7.

4. Sauro S, Thompson I, Watson TF. Ultra-morphology and dentine permeability changes induced by prophylactic procedures on exposed dentinal tubules in middle dentine. Med Oral Pat Oral Cir Bucal 2011; 16:e1022-30.

5. Tian L, Peng C, Shi Y, Guo X, Zhong B, Qi J et al. Effect of Mesoporous Silica Nanoparticles on Dentinal Tubule Occlusion: An In Vitro Study Using SEM and Image Analysis. Dent Mater J 2014; 33: 125-32.

6. Naghiu MA, Gorea M, Mutch E, Kritaly F, Tomoaia-Cotisel M. Forsterite Nanopowder: Structural characterization and biocompatibility evaluation. J. Mater Sci. Technol 2013; 29: 628-32.

7. Forghani A, Mapar M, Kharaziha M, Fathi MH. Novel fluroapatite- forsterite nanocomposite powder for oral bone defects. Int J Appl Cer Tech 2012; 10: 282-9.

8. Sayyedan FS, Fathi MH, Edris H, Doostmohammadi A, Mortazavi V, Shirani F. Fluoride release and bioactivity evaluation of glass ionomer: Forsterite nanocomposites. Dent Res J 2013; 10: 452-9.

9. Gillam D, Mordan N, Newman H. The Dentin Disc Surface: A Plausible Model for Dentin Physiology and Dentin Sensitivity Evaluation. Adv Dent Res 1997; 11:487-501.

10. Singh K, Naik R, Hegde S, Damda A. Shear Bond Strength of Superficial, Intermediate and Deep Dentin In Vitro with Recent Generation Self-etching Primers and Single nano Composite Resin. J Int Oral Health 2015; 7:28-32.
11. Barakat OA. The contribution of bonding agents, dentin desensitizing and deprotinizing agents on permeability and bond strength of dentin. PHD thesis, Cairo University, 2005.

12. El- Salamouni M. The effect of three desensitizing agents on dentin permeability at different depths. Master Thesis, Faculty of Dental Medicine for Girls, Al-Azhar University, 2012.

13. Niazy MA, Jamil WE. The Role of Adhesive Systems and Dentin Desensitizers in Altering the Permeability of Human Dentin. Cairo Dent J 2009; 25: 433-42.

14. Nabian N, Delavar M, Rabiee SM, Jahanshah M. Quenched/unquenched nanobioactive glass-ceramics: synthesis and in vitro bioactivity evaluation in ringer's solution with BSA. CI \& CEQ 2013; 19: 231-9.

15. Mohammadi M, Hesaraki S, Ardakani S. Comparative Study of in-vitro Behavior of Tetracalcium Phosphatebased Cement: Ringer's Solution versus Human Blood Plasma. IJEPR 2014; 3.

16. Torres C, Silva T, Fonseca B, Sales A, Holleben P, Di Nicolo R, Borges A. The Effect of Three Desensitizing Agents on Dentin Hypersensitivity: A Randomized, Splitmouth Clinical Trial. Op Dent 2014; 39:186-94.

17. Zhong Y, Liu J, Li X, Yin W, He T, Hu D et al. Effect of a novel bioactive glass-ceramic on dentinal tubule occlusion: an in vitro study. Aust Dent J 2015; 60: 96-103.

18. Ma Q, Wang T, Meng Q, Xu X, Wu H, Xu D, Chen Y. Comparison of in vitro dentinal tubule occluding efficacy of two different methods using a nano-scaled bioactive glasscontaining desensitizing agent. J Dent 2017; 60:63-9.

19. Tagami J, Sugizaki J, Hosoda H. Effects of the various pre treatments for dentine bonding on the dentine permeability. Japanese J Soci Dent Mater Dev. 1990; 9:240-46.

20. Farooq I, Imran Z, Farooq U, Leghari A, Ali H. Bioactive glass: a material for the future. World J Dent 2012; 3:199-201.

21. Rahman MN, Day AE, Bal BS, Fu Q, Jung SB, Bonewald LF. Bioactive glass in tissue engineering. Acta Biomater 2011; 7:2355-73.

22. Hiller KA, Buchalla W, Grillmeier I, Neubauer C, Schmalz $\mathrm{G}$. In vitro effects of hydroxyapatite containing toothpastes on dentin permeability after multiple applications and ageing. Scientific Reports 2018; 8:4888.

23. Vollenweider M, Brunner T, Knecht S, Grass RN, Zehnder $\mathrm{M}$, Imfeld $\mathrm{T}$ et al. Remineralization of human dentin using ultrafine bioactive glass particles. Acta Biomater 2007; 3:936-43. 
24. Earl JS, Wood DJ, Milne SJ. Nanoparticles for Dentine Tubule Infiltration: an In Vitro Study. J Nanosci Nanotechnol 2008; 9: 6668-74.

25. Chiang YC, Chen HJ, Liu HC, Kang SH, Lee BS, Lin FH et al. A Novel Mesoporous biomaterial for treating Dentin Hypersensitivity. J Dent Res 2010; 89:236-40.

26. Curtis AR, West NX, Su B. synthesis of nano-bioglass and formation of apatite rods to occlude exposed dentin tubules and eliminate hypersensitivity. Acta Biomater 2010; 6:3740-6.

27. Du MQ, Bian Z, Jiang H, Greenspan D.C, Burwell AK, Zhong J. Clinical evaluation of a dentifrice containing calcium sodium phosphosilicate (novamin) for the treatment of dentin hypersensitivity, Am J Dent 2008; 21:210-14.

28. Wang ZJ, Sa Y, Sauro S, Chen H, Xing WZ, Ma X. Effect of desensitising toothpastes on dentinal tubule occlusion: A dentine permeability measurement and SEM in vitro study. J Dent 2010; 38:400-10.

29. Pradeep AR, Sharma A. Comparison of clinical efficacy of a dentifrice containing calcium sodium phosphosilicate to a dentifrice containing potassium nitrate and to a placebo on dentinal hypersensitivity: a randomized clinical trial. J Periodontol 2010; 81:1167-73.

30. Pradeep AR, Agarwal E, Naik SB, Bajaj P, Kalra N. Comparison of efficacy of three commercially available dentifrices on dentinal hypersensitivity: a randomized clinical trial. Aust Dent J 2012; 57:429-34.

31. Ni S, Chou L, Chang J. Preparation and characterization of Forsterite (Mg2SiO4) bioceramics. Ceram Int 2007; $33: 83-8$.

32. Kharaziha M, Fathi M.H. synthesis and characterization of bioactive Forsterite nano-powder. Ceram Int 2009; 35:2449-54.

33. Besinis A, Noort R, Martin N. Infiltration of demineralized dentin with silica and hydroxyl apatite nanoparticles. Dent Mater 2012; 28:1012-23.

34. Besinis A, Noort RV, Martin N. Dentin infiltration with nanoparticles to promote remineralisation. Conference: British Society for Dental Research 2009.

35. Furtos G, Naghiu MA, Declercq H, Gorea M, Prejmerean C, Pana $\mathrm{O}$ et al. Nano Forsterite biocomposites for medical applications: Mechanical properties and bioactivity. J Bio Mater Res: Appl Biomater 2016; 104B: 1290-301.

36. Akatsuka R, Ishihata $\mathrm{H}$, Noji M, Matsumura $\mathrm{K}$, Kuriyagawa T, Sasaki k. Effect of hydroxyapatite film formed by powder jet deposition on dentin permeability. Euro J Oral Sci 2012; 120: 558-62.

37. Fahmy OM, Niazy MA, Gaweesh AS. Evaluation of the clinical effectiveness of four commercial in- office dentin desensitizers. Al-Azhar D J 2005; 8:209- 21.

38. Borges $\mathrm{AB}$, Barcellos $\mathrm{DC}$, Torres $\mathrm{CR}$, Borges AL, Marsilio AL, Carvahlo CA. Dentin hypersensitivity: etiology, treatment possibilities and other related factors: A literature review. World J Dent 2012; 3:60-7.

39. Sauro S, Pashley DH, Montanari M, Chersoni S, Carvalho RM, Toledano M. Effect of simulated pulpal pressure on dentin permeability and adhesion of self-etch adhesives. Dent Mater 2007; 23:705-13.

40. Orucoglu H, Belli S. Evaluation of the effect of four selfetching adhesives on dentin permeability. J Biomed Mater Res: Appl Biomater 2009; 90B:110-5.

41. Sayyedan FS, Fathi MH, Edris H, Doostmohammmadi A, Mortazavi V, Hanifi A. Effect Forsterite nano-particles on mechanical properties of glass ionomer cements. Ceram Int 2014; 40:10743-8

42. Bohner M. Can bioactivity be tested in vitro with SBF solution?. Biomater 2009; 30:2175-9.

43. Kulal R, Jayanti I, Sambashivaiah S, Bilchodmath S. An In-vitro Comparison of Nano Hydroxyapatite, Novamin and Proargin Desensitizing Toothpastes - A SEM Study. J Clin Diagn Res 2016; 10: ZC51-4.

44. Pashley DH. Mechanisms of dentin sensitivity. Dental Clinics of North America. 1990; 34:449-73.

45. Wang ZJ, Sa Y, Sauro S, Chen H, Xing WZ, Ma X. Effect of desensitising toothpastes on dentinal tubule occlusion: A dentine permeability measurement and SEM in vitro study. J Dent 2010; 38:400-10.

46. Sauro S, Watson TF. Dentin desensitization induced by prophylactic and air-polishing procedures: an in vitro dentin permeability and confocal microscopy study. J Dent 2010; 38:411-22.

47. Sales-Peres SH, Carvalho SN. Effect of propolis gel on the in vitro reduction of dentin permeability. J Appl Oral Sci 2011; 19:318-23.

48. Yilmaz N.A, Ertas E, Orucoğlu H. Evaluation of Five Different Desensitizers: A Comparative Dentin Permeability and SEM Investigation in Vitro. Open Dent J 2017; 11:15-33.

49. Stewardson DA, Crisp RJ, McHugh S, Lendemann U, Burke FJ. The effectiveness of system p- desensitizer in 
the treatment of dentin hypersensitivity. Prim Dent Core 2004 ; 11: 71-6.

50. Yu X, Liang B, Jin X, Fu B, Hannig M. Comparative in vivo study on the desensitizing efficacy of dentin desensitizers and one-bottle self etching adhesives. Op Dent 2010; 35 : 279-86.

51. Farghal NS, Abdalla AI, El-Shabrawy SM, Showaib EA. The effect of combined application of new dentin desensitizing agent and de-proteinization on dentin permeability of different adhesive systems. Tanta Dent J 2013; 10:138-44.

52. Madruga M, Silva A, Rosa W, Piva E, Lund R. Evaluation of dentin hypersensitivity treatment with glass ionomer cements: A randomized clinical trial. Braz Oral Res 2017; 31:e3.

53. Lenzi T, Guglielmi A, Arana-Chavez V, Raggio D. Tubule Density and Diameter in Coronal Dentin from Primary and Permanent Human Teeth. Microsc Microanal 2013; 9:1445-9. 